\title{
O uso do ácido hialurônico na harmonização facial: Uma revisão de literatura
}

\author{
The use of Hyaluronic Acid in facial harmonization: A literature review \\ El uso de Ácido Hialurônico em la armonización Facial: Uma revisión de la literatura \\ José Milton de Aquino e Silva Neto ${ }^{1 \star}$, Jordana Lima Vicente da Silva ${ }^{1}$, Altamiro Júnio Paranhos \\ Cesar de Mendonça ${ }^{1}$, Isabel Karine Ferreira Duarte ${ }^{1}$, João Francisco Tenório Neto'1.
}

\section{RESUMO}

Objetivo: Demonstrar a importância do ácido hialurônico $(\mathrm{AH})$, como sendo uma técnica utilizada não só para fins estéticos, como também restabelecer a parte funcional. Métodos: Tratou-se de uma revisão de literatura, com abordagem descritiva com caráter informativo, onde o processo de formulação se deu através de buscas por literaturas cientificas, em bases de dados, tendo como descritores: A importância do ácido hialurônico e seus benefícios nos dias atuais. Resultados: Nos últimos anos a utilização do ácido hialurônico, tem se destacado nos procedimentos referentes a harmonização facial, do qual é observado um significante resultado do melhoramento nas depressões e estruturas faciais, diminuindo consideravelmente os sinais de envelhecimento. Dentro desse âmbito é destacado inúmeros aspectos positivo referentes a esse polissacarídeo. Considerações Finais: a técnica de preenchimento é indolor uma vez que o paciente é submetido à anestesia local e em questão de minutos pode retomar suas atividades cotidianas sendo bastante eficaz e seguro para o retardo do envelhecimento precoce, melhorando significativamente as linhas de expressão e atenuando a assimetria facial.

Palavras-chave: Ácido hialurônico, Uso terapêutico, Preenchedores Dérmicos, Sistema tegumentar.

\begin{abstract}
Objective: To demonstrate the importance of hyaluronic acid (HÁ) as a technique used not only for aesthetic purposes, but also to restore the functional part. Methods: This was a review of the literature, with a descriptive approach with an informative character, where the formulation process took place through searches by scientific literature, in databases, having as descriptors: the importance of hyaluronic acid and its benefits in current days. Results: In recent years the use of hyaluronic acid has been highlighted in the procedures related to facial harmonization, which is a significant result of the improvement in depressions and facial structures, considerably reducing the signs of aging. Within this scope, many positive aspects regarding this polysaccharide are highlighted. Final Considerations: The filling technique is painless since the patient undergoes local anesthesia and in a matter of minutes can resume his daily activities being quite effective and safe for the delay of the early aging, significantly improving the lines of expression and attenuating the facial asymmetry.
\end{abstract}

Key Words: Hyaluronic Acid, Therapeutic use, Dermal fillers, Integumentary System.

\section{RESUMEN}

Objetivo: Demostrar la importancia del ácido hialurónico $(\mathrm{AH})$ como técnica utilizada no solo com fines estéticos, sino también para restaurar la parte funcional. Métodos: Esta fue uma revisión de la literatura, com

1Centro Universitário CESMAC (CESMAC), Maceió-Alagoas. *E-mail: milton_neto_166@hotmail.com

SUBMETIDO EM: 7/2019

ACEITO EM: 8/2019

PUBLICADO EM: $9 / 2019$ 
um enfoque descriptivo com um caráter informativo, donde el processo de formulación se llevó a cabo mediante búsquedas em la literatura científica, em bases de datos, teniendo como descriptores: La importancia del ácido hialurónico y sus benefícios en días actuales. Resultados: En los últimos años, el uso de ácido hialurónico se há destacado em los procedimentos relacionados con la armonización facial, que muestra un resultado significativo de la mejora en las depresiones y las estructuras gaciales, reduciendo considerablemente los signos de envejecimiento. Dentro de este alcance, se destacan muchos aspectos positivos con respecto a este polisacárido. Consideraciones finales: La técnica de llenado es indolora, ya que el paciente se somete a anestesia local y, em cuestión de minutos, puede reanudar sus atividades diárias siendo bastante efectivo y seguro para el retraso del envejecimiento prematuro, mejorando significativamente las líneas de expresión y atenuando la asimetría facial.

Palabras Clave: Ácido hialurónico, Uso terapêutico, Rellenos dérmicos, Integumento común.

\section{INTRODUÇÃO}

Nas últimas décadas a sociedade cada vez mais se preocupa não só no que se refere ao corpo como também a beleza facial, todavia, o processo de envelhecimento acontece de forma natural. Com o aumento da expectativa de vida eleva-se também o crescimento de buscas para melhorar os aspectos faciais, isso ocorre porque com o avanço da idade é possível observar o envelhecimento da pele, principalmente na face; tornando-se desta forma um dos motivos que levam as pessoas a buscarem recursos estéticos para diminuírem os efeitos do tempo (FERREIRA NR e CAPOBIANCO MP, 2016).

A pele é o maior órgão que envolve todo o corpo onde determina os limites com o meio externo, podendo exercer inúmeras funções: reguladora térmica, possui defesa orgânica contra microrganismos, exerce função no controle do fluxo sanguíneo e uma das mais importantes características é que possui funções sensoriais (calor, frio, pressão, dor e tato), sendo desta forma um órgão vital e sem ela, não existiria sobrevivência (SCHNEIDER LV, 2000).

Atualmente a Harmonização Facial vem crescendo com suas novas técnicas buscando como enfoque harmonizar a face de forma mais natural possível, deixando suas modificações de forma discretas, buscando sempre atender as expectativas e reduzir os sinais de envelhecimento. Os Cirurgiões Dentistas através de especializações podem atuar nessa área, tendo em vista que é um tratamento multidisciplinar (RIOS M, 2017).

Cada vez mais busca-se utilizar substâncias com o intuito de melhorar a estética facial, a princípio, as substâncias que era usada apresentavam pigmentos que possuíam extrações de origem vegetal e animal, onde eram aplicados topicamente nas pessoas com o propósito da melhoria da aparência (MONTEIRO EO, 2011).

Com o avanço das tecnologias e dos procedimentos o Cirurgião Dentista nunca esteve tão em evidência como nos dias atuais, principalmente devido ao grande aumento dos seus trabalhos em redes sociais serem divulgados. A aplicação de preenchedores como o Ácido Hialurônico (AH) possui indicações, além do aspecto estético e funcional, sendo coadjuvantes em alguns tratamentos como a distonia e volume facial (RIOS M, 2017).

Nesse contexto, é de suma importância que o profissional da Odontologia possua um bom senso e entendimento não só da anatomia facial como também da necessidade e expectativa de cada paciente, sem poder contraindicar a devida importância do tratamento multidisciplinar e possíveis necessidades nas indicações de outros profissionais ao paciente (GOODMAN GJ, et al., 2015).

As alterações estruturais da face são ocasionadas devido o envelhecimento, embora sejam habituais, são capazes de serem retardadas ou tratadas, como por exemplo, com a utilização do $A H$, que é um polissacarídeo que possui grande peso molecular, fornecido especialmente por fibroblastos e outras células que são especializadas do tecido conjuntivo. Levando em consideração que o mesmo é distribuído por todo o corpo, mas com o aumento da idade ocorre a degradação e diminuição da síntese do AH (GARBUGIO AF e FERRARI GF, 2010). 
Uma das grandes consequências na diminuição do AH é a perda da flexibilidade e elasticidade da pele, que por sua vez ocorre a formação de rugas e desidratação. Com o propósito de minimizar tais alterações, tem ocorrido o grande aumento em busca desses procedimentos e um dos principais motivos é que eles não são invasivos ou minimamente invasivos, onde vem ajudando e colaborando para o tratamento e suavização dos diversos tipos de rítides faciais. Já que este ácido é uma substância que o corpo absorve de forma benéfica, sendo mais utilizados na prática clínica para preenchimento de rugas e sulcos. Com a devida aplicação, o $\mathrm{AH}$ se adequa aos contornos do rosto, tratando as rugas e recuperando o volume e a hidratação natural da pele que foram perdidas ao logo dos anos (GARBUGIO AF e FERRARI GF, 2010).

Baseado nesses aspectos, o presente estudo tem como principal objetivo realizar uma revisão de literatura sobre a utilização do AH injetável pelos Cirurgiões Dentistas no tratamento de rejuvenescimento facial, bem como suas propriedades, principais efeitos e benefícios, tempo de duração e os possíveis efeitos colaterais do mesmo.

\section{METODOLOGIA}

Para o desenvolvimento deste trabalho foi realizada a metodologia qualitativa, sendo feita entre o período de janeiro de 2019 a junho de 2019, onde analisou-se artigos publicados em bases de dados eletrônicos (Pubmed, Mesh, portal de periódicos CAPES, EBSCO, DeCS). Foram consultados textos em língua portuguesa, inglesa e espanhola, que se referiram aos protocolos clínicos da utilização do ácido hialurônico, tendo como critérios de exclusão os períodos de publicações e os artigos que não estavam na integra, sendo selecionados os textos a partir do ano 2000 até o ano de 2019, dispondo dos seguintes descritores: Preenchimento, ácido hialurônico na estética.

\section{RESULTADOS E DISCUSSÃO}

O tecido epitelial é uma proteção que proporciona uma cobertura da superfície para o meio interno do corpo humano, ela possui o aspecto de ser impermeável com grande resistência onde possui uma alta flexibilidade no corpo que se uni com as membranas de revestimento. Um dos aspectos mais importantes da pele é que possui um órgão sensitivo com uma infinidade de terminações nervosas que fornecem sensibilidades e estímulos dolorosos. Entre suas funções principais é a preservação da homeostasia no organismo, uma fonte que regula a temperatura corpórea, excreção, sensibilidade a estímulos táteis, térmicos e dolorosos, possuindo uma capacidade de restabelecer e renovar a reparação tecidual, onde ocorre também a produção da vitamina D (BROMMONSCHEKEL J, et al., 2014).

Em uma análise utilizando termos anatômicos, o tecido epitelial é composto principalmente por dois tecidos justapostos, que incluem a epiderme e a derme, além da hipoderme. A camada mais superficial chamada de epiderme em geral é caracterizada pela constituição de quatro a cinco camadas ou estratos, cabido ao fato da camada lúcida estar ou não presente, sendo somente percebida em algumas amostras quando a pele se encontra espessa (BROMMONSCHEKEL J, et al., 2014).

Vasos sanguíneos, nervos, músculos eretores dos pelos e anexos cutâneos são encontrados na derme. Pode ser observado através de estudos que a pele é constituída por células e elementos extracelulares, que na maioria das vezes são residentes, como exemplo existe os fibroblastos, os histiócitos, os macrófagos e os mastócitos, além de existirem células migratórias como os leucócitos e os plasmócitos. Quanto aos elementos extracelulares, encontra-se as substâncias intersticiais e as fibras. Nas substâncias intersticiais, tem células e fibras de natureza variada. A substância fundamental é amorfa, sendo formada por glicoproteínas, especificamente mucopolissacarídeos ácidos, sendo observado que entre eles uns dos mais abundosos na derme é o AH (CROCCO El, et al., 2012).

A hipoderme ou tela subcutânea é a camada mais profunda, onde é estabelecida por um tecido adiposo que possui a função de possuir reservas de energia, onde ocorre o isolamento térmico dos organismos e a absorvência dos choques mecânicos. É importante ressaltar que esta camada de tecido adiposo, que se 
localiza abaixo da pele, diminui de forma bastante significativa com o aumento da idade (SILVA RM e ANDREATA MFG, 2017).

Em meados do ano 1934, foi iniciado estudos em busca de uma molécula versátil, o $\mathrm{AH}$. A análise para o respectivo estudo iniciou-se no Centro de Estudos de Bioquímica do Departamento de Oftalmologia da Universidade de Columbia, em que Karl Meyer e seus assistentes, expos um isolamento para essa substância, a partir do humor vítreo bovino, que até então era desconhecida. Nos anos posteriores, Meyer e colaboradores tiveram como foco isolar o AH presente na pele, sendo utilizados: articulações, cordão umbilical e crista de galo (GARBUGIO AF e FERRARI GF, 2010).

Em 1937, Kendall, Heidelberger e Dawson analisaram que existia uma grande semelhança entre um polissacarídeo da cápsula de bactérias do gênero Streptococcus do grupo A hemolítica e o $\mathrm{AH}$, sendo observado que sua origem era microbiana.

No entanto somente em 1950, Meyer e seus assistentes conseguiram definir a estrutura do AH possibilitando reconhecer e dominar as propriedades que nele existe. Tendo como resultado a existência de um polissacarídeo linear que possui grande massa molar onde consiste em elementos polianiônicos e dissacarídeos de ácido D-glucurônico (GlcUA) e Nacetilglicosamina (GlcNAc) unidos (GARBUGIO AF e FERRARI GF, 2010).

O resultado do envelhecimento intrínseco é bastante previsível, de forma inevitável, e essas alterações são diretamente relacionadas com tempo de vida. Uma das características mais marcantes da pele intrinsecamente envelhecida apresentam características como ressecamento, palidez, rugas finas, possui um certo nível de flacidez e algumas vezes possuem uma diversidade de tumores benignos. Algumas das características marcantes são os aspectos das bochechas que possuem aspectos cavados e a região orbicular oculares com flacidez que são causadas pela perda de gordura, com o ressecamento e a flacidez ocorre uma grande diminuição da ação das glândulas sebáceas, e perda de cabelo ou hirsutismo nas mulheres (SILVA RM e ANDREATA MFG, 2017).

Já quando se trata do envelhecimento extrínseco, ou foto envelhecimento, é ocorrido devido vários segmentos ocasionados pelo acumulativos de vários efeitos, e entre eles o que se destaca é a exposição crônica à radiação ultravioleta (UV), porém existem outras razões que acometem ainda mais a população como: poluição, radiação infravermelha, sedentarismo, alimentação, estresse, consumo de álcool e tabagismo (ALMEIDA ART e SAMPAIO GAA, 2016).

Na maioria das vezes os achados clínicos na pele foto envelhecida incluem rugas, algumas lesões que possuem pigmentações, sardas, lentigos e áreas de hiperpigmentação, e lesões despigmentastes, como hipomelanose gutata (BAUMANN L, 2004).

Os envelhecimentos ocasionados por radiação UV, apresentam relativas modificações que estimulam a aspereza e ressecamento do tecido epitelial, telangiectasia, rítides de grande profundidade e tumores cutâneos, incluindo as neoplasias malignas (GOODMAN GJ, et al., 2015).

Na matriz extracelular o AH é o glicosaminoglicano que possui maior abundancia na derme, onde não é sulfatado e não possui ligação covalente com a proteína, pois ele é o único glicosaminoglicano não limitado apenas a tecido animal, visto que pode encontrar-se também em cápsula de certas bactérias (AGOSTINI T e SILVA D, 2010).

Nos últimos anos o AH mais utilizado são os de origem não animal, onde são estabelecidos através de culturas bacterianas não patológicas, como é o caso do Streptococcus, essa forma de estabelecimento gera maior rendimentos com uma concentração mais elevada de $\mathrm{AH}$, sendo estabelecido com menor custo. Entre os estudos as bactérias, S. zooepidemicus é o que possui maior utilização. Nessa forma de obtenção de cultivo o AH possui características de um polissacarídeo extracelular sinteticamente parecido com o de animal, onde por sua vez pode-se obter um controle das características dos polímeros e com um menor custo benefício (GARBUGIO AF e FERRARI GF, 2010). 
Vários aspectos devem ser levado em consideração para a escolher o $\mathrm{AH}$ como: a análise da compatibilidade biológica, a segurança como irá ser aplicado no paciente, o equilíbrio no local da aplicação, possuir baixo risco de possíveis alergias, não possuir reações inflamatórias, não possuir substâncias que sejam carcinogênicas, não se transportar por fagocitose, possuir facilidade na aplicação, importante possuir aspecto o mais natural possível, baixa imunogenicidade, a procedência da obtenção do produto e a viabilidade do custo para o paciente. Estes são uns dos fatores analisados para ser utilizado de forma positiva com preenchedores dérmicos, visto que o $\mathrm{AH}$ atende todos os requisitos, tornando-o aceito na comunidade cientifica (FERREIRA NR e CAPOBIANCO MP, 2016).

$\mathrm{O}$ AH deve possuir massa molar adequada para sua aplicação, tendo que possuir um nível elevado de pureza, isso ocorre porque as moléculas que possuem um grande peso molecular podem provocar comportamentos de reações granulosas de corpo estranho, logo após a aplicação das injeções intradérmica. Visto esse fato das moléculas iniciais possuírem meia-vida e não sendo estabilizadas nas primeiras 24 horas, buscou-se melhorar essa técnica através do cross-linking, por meio dessa nova tecnologia ocorreu as ampliações e melhoramento da estabilidade e do tempo clínico do AH nos pacientes (CROCCO El, et al., 2012).

O procedimento feito com o $\mathrm{AH}$ não requer muitos cuidados especiais, ele não interfere no cotidiano e nem na rotina do paciente. Logo após sua aplicação pode ser utilizado gelo no local. Uma de suas contraindicações é buscar evitar exercícios físicos e exposição solar e outras fontes de calor (BORGES FS, 2006).

Em termos gerais a média recomendada para aplicação de AH em uma mesma área anatômica é de $1 \mathrm{ml}$, o máximo para uma boa segurança é de $2 \mathrm{ml}$. Aproximadamente em $80 \%$ dos pacientes submetidos a esse procedimento, $1 \mathrm{ml}$ do produto já é o bastante. Se no entanto for observado que essa quantidade não foi o suficiente para um bom resultado se faz uma nova aplicação em outra sessão, procurando desta forma obter um resultado esperado com uma segurança adequada (GOMES RK e GABRIEL M, 2006).

Em estudos feitos foram analisados que no nosso organismo a quantidade de AH são de 12 gramas, porém a grande maioria é localizada na pele, aproximadamente $7 \mathrm{~g}$, tendo como o principal objetivo dar volume, sustentação, hidratação e elasticidade a mesma; possuindo menor concentração no sangue (CROCCO El, et al., 2012).

Pretendendo obter maior e melhor conforto para o paciente, realiza-se uma boa anamnese e um prétratamento com anestésicos tópicos durante 30 minutos antes do procedimento. Antes do procedimento, é efetivado assepsias com clorexidina alcoólico a 4\%. Algumas marcas de AH contêm em seus componentes anestésico, como é o caso da lidocaína, sendo dessa forma dispensável o uso tópico de outros anestésicos (ALMEIDA ART e SAMPAIO GAA, 2016).

Observando-se as formas moleculares que são encontradas em sua composição, constata-se que sua consistência possui aspecto gelatinosa e densa, dessa maneira no tecido epitelial, especificamente na derme - AH procede como sendo um preenchedor de espaços, diminuindo possíveis choques, dando-lhe estabilização e colaborando com as propriedades elásticas por formar uma rede de estruturas helicoidais (AGOSTINI T e SILVA D, 2010).

Levando em consideração que tanto os procedimentos realizados com as aplicações de toxina botulínica como as de preenchedores faciais não são conceituados processos cirúrgicos; e levando em consideração o parecer estabelecido pela Comissão Especial, qualificada em elaboração de estudos quanto a alteração da atual resolução sobre as substancias acima mencionadas na Odontologia, confere a Lei no 4.324/64, regulamentada pelo Decreto no 68.704/71; resolve: Autorizar e regulamentar a utilização da toxina botulínica e dos preenchedores faciais pelo Cirurgião Dentista, com o propósito de atender pacientes de forma terapêuticas funcionais e/ou estéticas, desde que não exceda sua área anatômica dos seus respectivos desempenhos (BRASIL, 2018)

A exposição solar representa aproximadamente $80 \%$ do envelhecimento facial, sendo os radicais livres o fator principal (BAUMANN L, 2004), visto que eles são denominados como sendo células que através da 
utilização do oxigênio produzem energia e, nessa técnica, o oxigênio apresenta-se de forma instável sendo criado no decorrer das funções metabólicas mais básicas, como digestão e circulação. Outros fatores como a toxina encontrada nos pesticidas e a fumaça do cigarro e inúmeras poluições encontradas no meio ambiente geram também um grande número de radicais livres (GARBUGIO AF e FERRARI GF, 2010).

Com isso pode ser observado que o processo oxidativo, principalmente no que se refere as proteínas, gera uma modificação nos constituintes do tecido conectivo, como a redução das atividades proliferativas dos fibroblastos, desarranjo nas fibras de colágeno, diminuição da elastina e redução do AH (SILVA RM e ANDREATA MFG, 2017).

$\mathrm{Na}$ atualidade, o $\mathrm{AH}$ na forma de gel injetável possuem excelentes resposta na abordagem estética na correção das rítides, diminuição dos contornos e restituição do volume facial. Como amostra cita-se sua aplicação no preenchimento dos sulcos nasojugais, nos sulcos nasogenianos, na região periocular, na região da glabela, no aumento do volume labial, região malar, linha de marionete, mandibular, mento, pescoço e mãos, cicatriz, sendo usado também na rinomodelação. Levando sempre em consideração que o uso e o volume do AH que irá ser aplicado dependera das profundidades dos sulcos encontrados em cada paciente. (ALMEIDA ART e SAMPAIO GAA, 2016).

É observado que a composição do AH possui a mesma concentração independente da fonte, porém a conformidade das suas propriedades elásticas e viscosas estão diretamente concomitantes com sua massa molar. O AH é ligeiramente corrompido na derme e metabolizado no fígado, tendo como resultado a água e - CO2. Na proporção que ele é aplicado vai sendo degradado, contudo as moléculas de água buscam se ligar ao $\mathrm{AH}$ remanescente, levando ao processo de isovolumétria, isto é, mesmo com a absorção do preenchedor, ele continua sendo fator positivo no efeito cosmético, uma vez que há uma ascendência na ligação da água à trama de $\mathrm{AH}$ menos concentrada (AGOSTINI T e SILVA D, 2010).

Ao restabelecer o $\mathrm{AH}$ nas camadas epiteliais é reconstituída a compensação hídrica, onde é filtrado e regulado a disposição de proteínas na pele e constitui-se um ambiente físico em que ocorre a movimentação celular, com isso acontece a contribuição da melhoria no que se refere a estrutura e elasticidade da pele, suavizando as expressões faciais (FERREIRA NR e CAPOBIANCO MP, 2016).

O profissional deve fazer a anamnese individualmente por completo, avaliando possíveis antecedentes de alergias, e medicamentos, analisar benefícios e passar de forma clara para o paciente os possíveis riscos, além de debater com o paciente as expectativas esperadas. Importante requerer assinatura do paciente, no que se refere ao termo de consentimento e realizar fotografias antes e depois da aplicação do $\mathrm{AH}$. Quando necessário e com permissão escrita do médico, fazer o cancelamento temporário de anticoagulantes e antiinflamatórios não hormonais de sete a dez dias antes do procedimento, buscando dessa forma minimizar o aumento de sangramento, e após a técnica o paciente é aconselhado a minimizar esforços no local da aplicação e só tomar os medicamentos nos próximos dois dias, reduzindo desta forma possíveis hematomas (CROCCO El, et al., 2012).

Além disso, eventuais complicações também podem decorrer devido a inexperiência do profissional, utilização de técnica incorreta ou inerente até mesmo ao produto, levando em consideração suas diferentes formulações e concentrações (BISCHOFF JE, et al., 2000)

A maior área de risco é a glabela, devido possuir a capacidade de comprometimento vascular importante, podendo ocorrer efeitos como necrose tecidual e até cegueira. Além de que os feixes vasculares supratroclear e supraorbitario inervam a glabela. As causas de necrose tecidual nessa região pode ser ocasionado por injeção intravascular, constrição por grande quantidade de $\mathrm{AH}$ e/ou problemas vasculares, baixando o fornecimento do sangue no local (ALMEIDA ART e SAMPAIO GAA, 2016).

O Brasil, existem diversas marcas e tipos de AH comercializados e aprovados pela ANVISA. Embora todas tenham eficácia e segurança comprovadas cientificamente, por outro lado apresentam diferenças na composição. Por conseguinte, na duração, nas indicações aprovadas em bula e também na potência como pode ser demostrado na Quadro 1 (CONTOX, 2019). 
Quadro 1 - Principais preenchedores disponíveis no mercado brasileiro e suas indicações.

\begin{tabular}{|c|c|c|c|c|}
\hline NOMES COMERCIAIS & CARACTERISTICAS & & SUBTIPO/ATUAÇÃO & FABRICAÇÃO \\
\hline RENNOVA & $\begin{array}{l}\text { Gel viscoelástico e transparente } \\
\text { à base de } \mathrm{AH} \text {, origem não } \\
\text { animal, altamente biocompatível e } \\
\text { comprovadamente muito seguro. }\end{array}$ & Rennova Lift & $\begin{array}{l}\text { Repõe o volume perdido da face, realça o } \\
\text { perfil e cria lábios mais volumosos. } \\
\text { Elimina ou suaviza linhas e rugas. melhora } \\
\text { a hidratação, luminosidade e elasticidade, } \\
\text { atenua e pés de galinha. }\end{array}$ & $\begin{array}{l}\text { Croma Pharma GmbH, } \\
\text { sendo encontrado como: } \\
\text { Rennova Fill } \\
\text { Rennova Lift. }\end{array}$ \\
\hline PERFECTHA & $\begin{array}{l}\text { Utiliza fibras de hialuronato de } \\
\text { alto peso molecular e de alta } \\
\text { pureza, apresenta um ótimo } \\
\text { efeito volumizador e de longa } \\
\text { duração. preenchedor bifásico de } \\
\text { AH de fácil injeção. }\end{array}$ & $\begin{array}{l}\text { PERFECTHA } \\
\text { FINELINES } \\
\text { PERFECTHA DERM } \\
\text { PERFECTHA DEEP } \\
\text { PERFECTHA } \\
\text { SUBSKIN }\end{array}$ & $\begin{array}{l}\text { Revitalização cutânea, preenchimento de } \\
\text { microfissuras superficiais da face (terço médio } \\
\text { superior) } \\
\text { Contorno labial, código de barras, coxo lacrimal } \\
\text { Sulcos profundos, rinomodelação, volume } \\
\text { labial } \\
\text { volumização óssea (malar, zigomático, } \\
\text { contorno de mandíbula). }\end{array}$ & $\begin{array}{l}\text { Fabricado pela Sinclair } \\
\text { Pharma@ Sendo } \\
\text { encontrado como: } \\
\text { Perfectha Fine Line } \\
\text { Perfectha Derm }^{\mathrm{TM}} \\
\text { Perfectha Deep }^{\mathrm{TM}} \\
\text { Perfectha Subskin }^{\mathrm{TM}}\end{array}$ \\
\hline PRINCESS & $\begin{array}{l}\text { Gel isotônico e biodegradável, que } \\
\text { é usado para tratamento de } \\
\text { mesoterapia e correção de rugas } \\
\text { superficiais na camada superior } \\
\text { da pele. }\end{array}$ & $\begin{array}{l}\text { Princess Filler } \\
\text { Princess Volume } \\
\\
\text { Princess } \quad \text { Filler } \\
\text { Lidocaine. } \\
\text { Princess Plus. }\end{array}$ & $\begin{array}{l}\text { Corrige rugas e sulcos profundos, aumento ou } \\
\text { restauração do volume facial e dá forma aos } \\
\text { contornos faciais. Recomenda-se injetar o } \\
\text { Princess VOLUME na derme profunda ou } \\
\text { hipoderme. } \\
\text { Corrige rugas faciais moderadas a profundas e } \\
\text { aumento do volume dos lábios. Recomenda-se } \\
\text { injetar o Princess Filler na derme média a } \\
\text { profunda. } \\
\text { É indicado para corrigir o déficit e restaurar } \\
\text { substancialmente o volume facial, moldar os } \\
\text { contornos do rosto e auxiliar no tratamento } \\
\text { reconstrutivo de lipoatrofia facial, cicatrizes } \\
\text { debilitantes e assimetria morfológica. } \\
\text { Melhoria de Hidratação, tônus e elasticidade da } \\
\text { pele, correção das linhas superficiais e rugas } \\
\text { finas (pés de galinha, linhas de fumo). } \\
\text { Correção de rugas e sulcos profundos, } \\
\text { aumento de volume facial, remodelação dos } \\
\text { contornos faciais. }\end{array}$ & $\begin{array}{l}\text { fabricado pela croma- } \\
\text { pharma, } \\
\text { encontrado como: } \\
\text { Princess Rich, } \\
\text { Princess Filler, } \\
\text { Princess Volume, } \\
\text { Princess } \\
\text { Lidocaine. Filler } \\
\text { Princess Plus. }\end{array}$ \\
\hline
\end{tabular}




\begin{tabular}{|c|c|c|c|c|}
\hline TEOSYAL & $\begin{array}{l}\text { Preenchedores cutâneos injetáveis } \\
\text { de origem não animal e ácido } \\
\text { hialurônico biodegradável. }\end{array}$ & $\begin{array}{l}\text { TEOSYAL RHA } 1 \\
\text { TEOSYAL RHA } 2 \\
\text { TEOSYAL RHA } 3 \\
\text { TEOSYAL RHA } 4 \\
\text { TEOSYAL 27G DEEP } \\
\text { LINES } \\
\text { TEOSYAL 30G } \\
\text { GLOBAL ACTION }\end{array}$ & $\begin{array}{l}\text { Especificamente para rugas finas e superficiais } \\
\text { para reduzir rugas em áreas móveis em longo } \\
\text { prazo. } \\
\text { Deve ser usado especificamente para rugas } \\
\text { moderadas em rugas médias a profundas. } \\
\text { Baseado em hialurônico e deve ser usado } \\
\text { especificamente para rugas profundas na } \\
\text { derme profunda. } \\
\text { Usado especificamente para volume em áreas } \\
\text { extensas como a derme profunda à área } \\
\text { subcutânea. } \\
\text { Usado para tratar sulcos nasais, contornos } \\
\text { faciais nas bochechas e linha do maxilar e } \\
\text { aumento dos lábios. } \\
\text { Usado para a correção de rugas leves a } \\
\text { moderadas, linha glabelares, linhas nasolabiais } \\
\text { e áreas periorbitais e periorais }\end{array}$ & $\begin{array}{l}\text { Fabricado pela Noedic } \\
\text { Medical Solutions sendo } \\
\text { encontrado como os mais } \\
\text { relevantes: } \\
\text { TEOSYAL RHA } 1 \\
\text { TEOSYAL RHA } 2 \\
\text { TEOSYAL RHA } 3 \\
\text { TEOSYAL RHA } 4 \\
\text { TEOSYAL 27G DEEP } \\
\text { LINES } \\
\text { TEOSYAL 30G GLOBAL } \\
\text { ACTION }\end{array}$ \\
\hline JUVEDERM & $\begin{array}{l}\text { Usados para rapidamente } \\
\text { suavizar rugas moderadas a } \\
\text { severas como os vincos } \\
\text { nasolabiais. Pode também ser } \\
\text { usado para aumentar os lábios e } \\
\text { reparar a perda de volume para } \\
\text { uma aparência mais fresca e } \\
\text { juvenil, usando a tecnologia } \\
\text { hylacross para permitir um gel } \\
\text { mais suave e maleável. }\end{array}$ & $\begin{array}{l}\text { JUVEDERM } \\
\text { HYDRATE } \\
\text { JUVEDERM VOLUMA } \\
\text { LIDOCAINE } \\
\text { JUVEDERM ULTRA } \\
\text { SMILE } \\
\text { JUVEDERM VOLIFT } \\
\text { LIDOCAINE }\end{array}$ & $\begin{array}{l}\text { Usado para a derme superficial a média } \\
\text { tratando linhas finas e especialmente } \\
\text { aquelas ao redor dos cantos dos olhos e } \\
\text { aquelas bem próximas à superfície da } \\
\text { pele. } \\
\text { Usado na derme média a profunda para } \\
\text { suavizar rugas médias e profundas entre o } \\
\text { nariz e cantos da boca e ao redor dos } \\
\text { lábios. } \\
\text { Usado para preencher a derme profunda e } \\
\text { é uma formulação robusta altamente } \\
\text { interligada para dar volume e corrigir } \\
\text { vincos e rugas profundas, incluindo o } \\
\text { aumento do volume das bochechas e } \\
\text { queixo. } \\
\text { Age como um agente hidratante, atraindo } \\
\text { a umidade, preservando a pele para } \\
\text { manter um brilho saudável. }\end{array}$ & $\begin{array}{lr}\text { Fabricado por Nordic } \\
\text { Medical Solutions ApS } \\
\text { sendo encontrado como: } \\
\text { JUVEDERM ULTRA 2; } \\
\text { JUVEDERM ULTRA 3; } \\
\text { JUVEDERM ULTRA 4; } \\
\text { JUVEDERM HYDRATE; } \\
\text { JUVEDERM VOLUMA } \\
\text { LIDOCAINE; } \\
\text { JUVEDERM } \\
\text { SMILE; } \\
\text { JUVEDERM } \\
\text { LIDOCAINE; VOLIFT } \\
\text { JUVEDERM } \\
\text { RETOUCH VOLIFT } \\
\text { LIDOCAINE } \\
\text { JUVEDERM VOLBELLA } \\
\text { LIDOCAINE; }\end{array}$ \\
\hline
\end{tabular}




\begin{tabular}{|c|c|c|c|c|}
\hline & & $\begin{array}{lr}\text { JUVEDERM } & \text { VOLIFT } \\
\text { RETOUCH } & \text { W. } \\
\text { LIDOCAINE } & \\
\text { JUVEDERM } & \\
\text { VOLBELLA } & \\
\text { LIDOCAINE } & \\
\text { JUVEDERM } & \text { VOLITE } \\
\text { LIDOCAINE } & \end{array}$ & $\begin{array}{l}\text { Efeito de volume que recontorna e adiciona } \\
\text { volume ao queixo, bochechas e maçãs do rosto } \\
\text { e proporciona restauração duradoura dos } \\
\text { volumes faciais. } \\
\text { Juvederm Ultra Smile é usado para } \\
\text { aumentar o volume labial, redefinir o } \\
\text { contorno labial, tratar linhas labiais } \\
\text { verticais e depressões nos cantos da boca. } \\
\text { É projetado para suavizar rugas profundas } \\
\text { e vincos nasolabiais para uma aparência } \\
\text { renovada, assim como restaurar } \\
\text { suavemente os contornos naturais da } \\
\text { parte de baixo do rosto. } \\
\text { Usado para ajudar a tratar depressões } \\
\text { profundas na pele assim como restaurar o } \\
\text { volume e aumentar o contorno facial. } \\
\text { Utilizando a tecnologia exclusiva VYCROSS TM } \\
\text { para suavemente aumentar o formato dos } \\
\text { lábios ou restaurar o volume perdido. } \\
\text { Um tratamento injetável projetado para } \\
\text { melhorar a qualidade da pele por até } 9 \\
\text { meses. Juvederm Volite deve ser usado no } \\
\text { rosto, pescoço, decote e mãos. }\end{array}$ & $\begin{array}{l}\text { JUVEDERM } \\
\text { LIDOCAINE. }\end{array}$ \\
\hline HIALUROX & $\begin{array}{l}\text { O Hialurox® Ultra Soft é um gel } \\
\text { transparente, apirogénico, } \\
\text { fisiológico de Ácido Hialurônico } \\
\text { reticulado de origem não animal, } \\
\text { esterilizado através de calor } \\
\text { úmido. }\end{array}$ & $\begin{array}{l}\text { Ultra Soft } 8 \mathrm{mg} / \mathrm{g} \\
\text { Ultra Fine } 16 \mathrm{mg} / \mathrm{g} \\
\text { Ultra Fill } 24 \mathrm{mg} / \mathrm{g} \\
\text { Ultra Lift } 26 \mathrm{mg} / \mathrm{g}\end{array}$ & 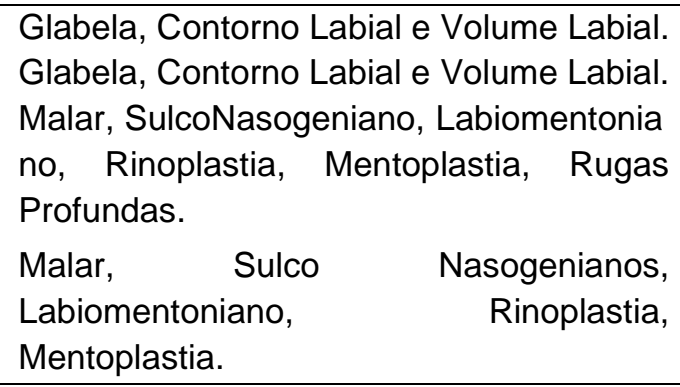 & $\begin{array}{l}\text { Fabricado por Hialurox } \\
\text { Preenchedores sendo } \\
\text { encontrado como: } \\
\text { Ultra Soft } 8 \mathrm{mg} / \mathbf{g} \text {; } \\
\text { Ultra Fine } 16 \mathrm{mg} / \mathbf{g} \text {; } \\
\text { Ultra Fill } 24 \mathrm{mg} / \mathbf{g} \text {; } \\
\text { Ultra Lift } 26 \mathrm{mg} / \mathbf{g} \text {. }\end{array}$ \\
\hline
\end{tabular}




\begin{tabular}{|c|c|c|c|c|}
\hline BELOTERO & $\begin{array}{l}\text { Gel viscoelástico e transparente } \\
\text { à base de } \mathrm{AH} \text {, origem não } \\
\text { animal, altamente biocompatível e } \\
\text { comprovadamente muito seguro. }\end{array}$ & $\begin{array}{l}\text { BELOTERO }{ }^{\circledast} \text { Soft } \\
\text { Lidocaine } \\
\text { BELOTERO }{ }^{\circledast} \text { Balance } \\
\text { Lidocaine } \\
\\
\text { BELOTERO }{ }^{\circledast} \text { Intense } \\
\text { Lidocaine } \\
\text { BELOTERO }{ }^{\circledast} \text { Volume } \\
\text { Lidocaine }\end{array}$ & $\begin{array}{l}\text { Usado na correção de pés de galinha } \\
\text { Linhas periorais, suaves (código de } \\
\text { barras), Linhas finas da testa, Linhas } \\
\text { suaves entre as sobrancelhas } \\
\text { Usados em bigode chinês moderado Linhas de } \\
\text { Marionete, moderadas Linhas periorais, } \\
\text { moderadas (código de barras), Linhas } \\
\text { moderadas entre as sobrancelhas, Contorno } \\
\text { dos lábios, Pés de Galinha, Filtro Labial, } \\
\text { Olheira. } \\
\text { Bigode chinês profundo, Linhas de } \\
\text { Marionete profundas, Volume Labial } \\
\text { Maçãs do rosto, Preenchimento do queixo, } \\
\text { Têmporas. }\end{array}$ & 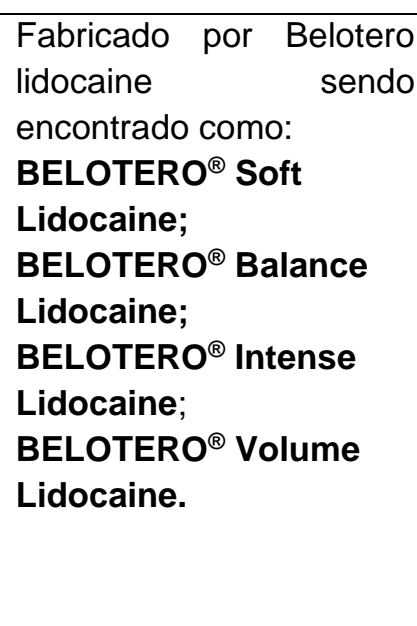 \\
\hline VARIODERM & $\begin{array}{l}\text { Mais alto padrão, o mercado de } \\
\text { preenchedores de tecidos moles e } \\
\text { re-absorvíveis, oferecendo uma } \\
\text { solução segura, eficiente e de } \\
\text { longa duração. }\end{array}$ & $\begin{array}{l}\text { VARIODERM Fineline } \\
\text { VARIODERM Basic } \\
\text { VARIODERM Plus } \\
\text { VARIODERM } \\
\text { Subdermal } \\
\text { VARIODERM Lips \& } \\
\text { Médium }\end{array}$ & $\begin{array}{l}\text { Rugas superficiais, pés de galinha, linhas } \\
\text { de periorbitais e levantamento de } \\
\text { sobrancelhas. } \\
\text { Rugas médias, contorno e volume labial. } \\
\text { Rugas profundas, sulcos nasogenianos e } \\
\text { comissuras orais. } \\
\text { Contornos da face e criação de volumes } \\
\text { faciais. } \\
\text { Excelente visco-elasticidade para as } \\
\text { regiões dos anteriores citados. }\end{array}$ & $\begin{array}{l}\text { Fabricado por Derma } \\
\text { Dream Corporation sendo } \\
\text { encontrado como: } \\
\text { VARIODERM Fineline; } \\
\text { VARIODERM Basic; } \\
\text { VARIODERM Plus; } \\
\text { VARIODERM } \\
\text { Subdermal; } \\
\text { VARIODERM Lips \& } \\
\text { Médium. }\end{array}$ \\
\hline $\begin{array}{l}\text { CIENTIFIC } \\
\text { HYALURONIC }\end{array}$ & $\begin{array}{l}\text { O Hialurox® Ultra Lift é um gel } \\
\text { transparente, apirogénico, } \\
\text { fisiológico de Ácido Hialurônico } \\
\text { reticulado, de origem não animal, } \\
\text { monofásico, esterilizado através } \\
\text { de calor úmido. }\end{array}$ & $\begin{array}{l}\text { Ultra Soft } 8 \mathrm{mg} / \mathrm{g} \\
\text { Ultra Fine } 16 \mathrm{mg} / \mathrm{g} \\
\text { Ultra Fill } 24 \mathrm{mg} / \mathrm{g} \\
\text { Ultra Lift } 26 \mathrm{mg} / \mathrm{g}\end{array}$ & $\begin{array}{l}\text { Palpebras, Contorno Labial, Perioculares, } \\
\text { Hidratação Intradermica e Mãos. } \\
\text { Palpebras, Contorno Labial, Volume Labial, } \\
\text { Perioculares. } \\
\text { Sulco Nasogeniano, Labiomentoniano, } \\
\text { Rinoplastia, Mentoplastia. } \\
\text { Malar, Sulco Nasogenianos, Rinoplastia, } \\
\text { Mentoplastia. Indicado a aplicação na derme } \\
\text { profunda. }\end{array}$ & $\begin{array}{l}\text { Fabricado por Hialumed } \\
\text { sendo encontrado como: } \\
\text { Ultra Soft } 8 \mathrm{mg} / \mathrm{g} \text {; } \\
\text { Ultra Fine } 16 \mathrm{mg} / \mathrm{g} \text {; } \\
\text { Ultra Fill } 24 \mathrm{mg} / \mathrm{g} \text {; } \\
\text { Ultra Lift } 26 \mathrm{mg} / \mathrm{g} \text {. }\end{array}$ \\
\hline
\end{tabular}

Fonte: Neto JMAS, Silva JLVS, Mendonça AJPC, 2019; (Baseado em CONTOX ®, 2019). 


\section{CONSIDERAÇÕES FINAIS}

Diante do exposto, alguns cuidados como uma boa vida saudável, uma boa hidratação, podem adiar as rugas. $E$ entre os procedimentos para precaução ou tratamento do envelhecimento dos tecidos epiteliais, citase o $\mathrm{AH}$, para a vigorarão da facial, cuja perda endógena reflete na redução da elasticidade, o que dá origem a flacidez e as rugas. O preenchimento é indolor visto que o paciente é submetido à analgesia local e em pouco tempo pode retomar suas atividades cotidianas exibindo apenas algum inchaço e vermelhidão no local da aplicação que desvanece naturalmente. Pode-se concluir que o preenchimento facial com ácido hialurônico é bastante eficaz e seguro, melhorando o aspecto de linhas de expressão e até mesmo remodelando expressões não condizentes com a simetria facial.

\section{REFERÊNCIAS}

1. AGOSTINI T, SILVA D. Ácido hialurônico: princípio ativo de produtos cosméticos. Santa Catarina, 2010.

2. ALMEIDA ART, SAMPAIO GAA. Ácido hialurônico no rejuvenescimento do terço superior da face: revisão e atualização - Parte 1. Surgical \& Cosmetic Dermatology, São Paulo, v.8, n.2, p.148-153, 2016.

3. BAUMANN L. Dermatologia cosmética: princípios e práticas. Rio de Janeiro: Revinter, 2004.

4. BISCHOFF JE, et al. Finite element modeling of human skin using an isotropic, nonlinear elastic constitutive model. J Biomech 2000;33(6):645-52.

5. BORGES FS. Dermato-funcional: modalidades terapêuticas nas disfunções estéticas. São Paulo: Phorte, 2006.

6. BRASIL. Conselho Federal de Odontologia (CFO). Resolução no 176/2018 de 04 maio. 2018. Nova redação aos artigos 3ำ e 4ํe e inclui os anexos VII e VIII da Resolução/CFO Decreto no 68.704/71. Disponível em: <http://www. http://cfo.org.br/website/nota-de-esclarecimento-2/>. Acesso em: 6 abr. 2019.

7. BROMMONSCHEKEL J, et al. Los efectos del ácido hialurónico en la prevención del envejecimiento cutáneo: una revisión de la literatura. EFDeportes.com, Revista Digital, Buenos Aires, año 19, n.192, Mayo de 2014.

8. CONTOX ®. 2019. In: Congresso Brasileiro de Toxina Botulínica e Preenchimento na Odontologia. Cuiabá: Disponível em: https://contox.com.br/9-marcas-de-acidos-hialuronicos-vendidas-no-brasil/. Acesso em: 08 jul. 2019.

9. CROCCO El, et al. Eventos adversos do ácido hialurônico injetável. Surgical \& Cosmetic Dermatology, São Paulo, v.4, n.3, p.259-263, 2012.

10. FERREIRA NR, CAPOBIANCO MP. Uso do ácido hialurônico na prevenção do envelhecimento facial. 2016.

11. GARBUGIO AF, FERRARI GF. Os benefícios do ácido hialurônico no envelhecimento facial. Revista UNINGÁ Review, Paraná, v.2, n.4, p.25-36, out. 2010.

12. GOMES RK, GABRIEL M. Cosmetologia: Descomplicando os Princípios Ativos. 1ª ed. São Paulo. Livraria Médica Paulista Editora, 2006.

13. GOODMAN GJ, et al. Current Concepts in the Use of Voluma, Volift, and Volbella.Plast Reconstr Surg. 2015 Nov; 136 (5):139S-148S.

14. MONTEIRO EO. Tratamento de rejuvenescimento facial com ácido hialurônico não estabilizado de origem não animal aplicado na derme. Moreira Jr. Editora; 198 a 200. Revista Brasileira de Medicina, v. 68, n 6, 2011

15. RIOS M. Harmonização orofacial: um novo conceito na odontologia. São Paulo: Artes Médicas, 2017

16. SCHNEIDER LV. Estrutura da Pele e seus Anexos. Curso Extensivo de Cosmetologia, Porto Alegre: v.1, n. 5, p.213, 2000.

17. SILVA RM, ANDREATA MFG. Rejuvenescimento facial: a eficácia da radiofrequência associada à vitamina $C$. Revista Maiêutica, Santa Catarina, v.1, n.1, p. 55-73, 2017. 